\title{
Influencia del sistema nitridérgico en la respuesta contráctil a fenilefrina de anillos de vasos usados en revascularización coronaria
}

\author{
Juan C Prieto ${ }^{1,2}$, G ianni Pinardi2, Jaime Zamorano ${ }^{1}$, \\ Ernesto Larraín ${ }^{1}$, Cristián Bermúdez ${ }^{1}$, Rodrigo Castillo ${ }^{1}$, \\ Verónica Cisternas $^{1 a}$, Hugo F Miranda ${ }^{2 b}$. \\ Modification of phenylephrine induced \\ contraction of human vessel rings by \\ L-arginine and L-arginine methyl ester
}

Background: Endothelial dysfunction is associated to a lower production of nitric oxide and a reduction of endothelium mediated vasodilation. Aim: To study the effects of pharmacological agents that modify nitric oxide synthetase (NOS) activity on tension changes induced by phenylephrine in rings of internal mammary and radial arteries and saphenous vein. Material and methods: Vessel rings of 7 to $10 \mathrm{~mm}$ length were obtained from 32 patients subjected to coronary vascular surgery. Fourteen samples of radial artery, 12 samples of internal mammary artery and 15 samples of saphenous vein were obtained. A maximal contraction was induced with $\mathrm{KCl}$ and dose response curves for phenylephrine (FE) in the absence or presence of L-arginine and L-arginine methyl ester (L-NAME), were constructed. Results: The tension induced by FE in internal mammary artery and saphenous vein reached a maximum, near $90 \%$ of $80 \mathrm{mM} \mathrm{KCl-induced} \mathrm{contraction,} \mathrm{but} \mathrm{in} \mathrm{the} \mathrm{radial} \mathrm{artery,} \mathrm{it} \mathrm{reached} \mathrm{a} \mathrm{maximum} \mathrm{of}$ $63 \%$ ( $\mathrm{p}<0.05$ ). In all vessels, the dose response curves were significantly shifted to the right by $\mathrm{L}-$ arginine and to the left by L-NAME. Conclusions: Pre-incubation of human rings with L-ARG or L-NAME, changed the response to FE induced contraction, which may be related to different degrees of endothelial nitric oxide production or NO sensitivity. The basal NO production in radial artery seems to be larger than the other vessels (Rev Méd Chile 2007; 135: 1231-36).

(Key words: Endothelium, vascular; Nitric oxide; Phenylephrine)

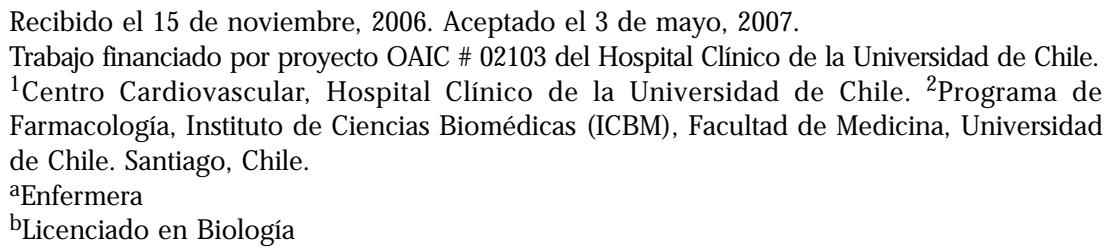

Correspondencia a: Dr. JC Prieto. Programa de Farmacología, ICBM,

Facultad de Medicina, Universidad de Chile. Independencia 1027,

Santiago 7, Chile. Teléfono: 56-2-978-6050. Fax: 56-2-737-2783.

E mail: jprieto@med.uchile.cl 
os vasos utilizados con mayor frecuencia en —cirugía coronaria, para confeccionar puentes en revascularización miocárdica, son la arteria mamaria interna (AMI), la vena safena (VS) y la arteria radial (AR). Sin embargo, la heterogeneidad funcional del endotelio en los distintos vasos puede ser determinante para el éxito tanto en el posoperatorio inmediato como el posoperatorio a largo plazo ${ }^{1}$. Generalmente, se prefieren los conductos arteriales por su mayor tasa de permeabilidad en el tiempo comparado con los confeccionados con venas, cuando el puente es efectuado en sitios anatómicos equivalentes ${ }^{2}$. La diferencia en la permeabilidad a largo plazo entre arterias y venas, se explicaría, entre otros factores, por características específicas de los conductos, tales como la producción endotelial de óxido nítrico (NO). Se acepta que la deficiencia de NO en enfermedades de arterias coronarias o periféricas es predictiva de futuros eventos cardiovascula$\mathrm{res}^{3,4}$. Además, la AR y la AMI tienen diferentes características biológicas, especialmente relacionadas con el grosor de la capa muscular y los niveles de producción de prostaciclinas ${ }^{5-7}$.

Las células endoteliales están relacionadas estrechamente con la regulación del tono vascular, inflamación, regulación de factores de crecimiento, trombolisis, lipolisis y antiagregación plaquetaria ${ }^{8}$. Muchas de estas funciones están asociadas con la producción endotelial de NO debido a la conversión de L-arginina a L-citrulina, proceso catalizado por la enzima constitutiva óxido-nitrosintasa endotelial (e-NOS o NOS III) ${ }^{1,9}$. El NO media la vasodilatación dependiente de endotelio, oponiéndose a los efectos de potentes vasoconstrictores derivados de endotelio tales como angiotensina II y endotelina ${ }^{4}$. La relajación de las células musculares lisas de los vasos depende de la activación de la guanilciclasa soluble y del incremento de $\mathrm{GMPc}^{10}$.

La disfunción endotelial es reconocida actualmente como un importante proceso en la patogénesis de la ateroesclerosis. Se manifiesta precozmente como resultado de la exposición de las células endoteliales a factores de riesgo cardiovascular tales como edad avanzada, tabaquismo, diabetes, hipertensión o dislipidemia ${ }^{11,12}$.

La disfunción endotelial se acompaña de una reducción en la producción de $\mathrm{NO}^{1,13}$ y una reducción crítica de la vasodilatación mediada por endotelio. El éster metílico de la $\mathrm{N}^{\mathrm{G}}$-nitro-Larginina (L-NAME), un inhibidor no selectivo de NOS in vivo e in vitro, es capaz de reducir significativamente la producción y liberación de $\mathrm{NO}^{14}$. Por otra parte, puesto que la L-arginina (LARG) es el precursor en la síntesis endógena de $\mathrm{NO}$, se ha demostrado que la administración aguda o crónica de L-arginina in vivo, mejora la respuesta vasodilatadora, probablemente por un incremento de la producción de $\mathrm{NO}^{15}$.

El objetivo del presente estudio fue evaluar el efecto de fármacos que modifican la actividad de la NOS, sobre los cambios de tensión inducidos por el agonista selectivo $\alpha 1$-adrenérgico fenilefrina (FE) en anillos de AMI, AR y VS humana. Al mismo tiempo, se determinó si existen diferencias en la respuesta contráctil entre los diferentes vasos empleados en revascularización coronaria.

\section{MATERIAL Y MÉTODO}

Recolección de muestras. Se usaron muestras de AR, $n=14$, AMI, $n=12$ y VS, $n=15$ obtenidas de 32 pacientes sometidos a cirugía de revascularización coronaria en el Hospital Clínico de la Universidad de Chile entre mayo de 2003 y junio de 2004. El protocolo de estudio fue aprobado por el Comité de Ética de la Facultad de Medicina y todos los pacientes firmaron el consentimiento informado. Se obtuvieron anillos de aproximadamente 7-10 $\mathrm{mm}$ de longitud de cada vaso, los cuales no eran sometidos a lavados con solución salina o vasodilatadores. Las muestras se mantuvieron en una solución Tyrode modificada con la siguiente composición, en $\mathrm{mM}$ : $\mathrm{NaCl} 119,7, \mathrm{KCl} 5,3, \mathrm{CaCl}_{2}$ 2,7, $\mathrm{MgSO}_{4} 1,2, \mathrm{KH}_{2} \mathrm{PO}_{4}, 1,2, \mathrm{NaHCO}_{3}$ 23,8, glucosa $11,1^{16}$, mantenida a $4^{\circ} \mathrm{C}$. Los anillos de vasos se prepararon en el laboratorio dentro de los $30 \mathrm{~min}$ después de su obtención.

Estudios vasculares. Los anillos fueron montados para su estudio in vitro en ganchos de acero inoxidable y colocados en baños para órganos de $25 \mathrm{~mL}$ con solución Tyrode mantenida a $37^{\circ} \mathrm{C}$, burbujeada continuamente con una mezcla de $95 \% \quad \mathrm{O}_{2} / 5 \% \mathrm{CO}_{2}$, tal como se ha descrito en trabajos previos ${ }^{16}$. Uno de los extremos se unió a un transductor Grass de fuerza desplazamiento (FT 0,3), que estaba conectado a un polígrafo 
Grass model 7 (Grass Instruments, Quincy, Mass, U.S.A.), destinado a registrar los cambios de tensión. El otro extremo estaba fijo al fondo interior del baño. Los anillos arteriales y venosos fueron estabilizados durante $60 \mathrm{~min}$, sometidos a una tensión basal de 1,5 y $1 \mathrm{~g}$, respectivamente, reemplazando la solución Tyrode cada 15 min.

Después del período de estabilización, los anillos fueron contraídos en dos ocasiones con una solución salina de $80 \mathrm{mM}$ de $\mathrm{KCl}$, hasta alcanzar la respuesta máxima y por sucesivos cambios del Tyrode se alcanzaba la línea basal. La mayor respuesta a $\mathrm{K}^{+}$, fue considerada como contracción basal. Después de obtenida la línea basal, se construyó una curva dosis respuesta a FE $\left(10^{-6}-10^{-3,5} \mathrm{M}\right)$ en dosis acumulativas de $\frac{1}{2}$ unidad logarítmica. Una vez obtenida la respuesta contráctil máxima, se procedió al lavado de la preparación con la solución Tyrode para alcanzar la tensión basal inicial.

Posteriormente se incubó durante $60 \mathrm{~min}$ ya sea con: a) $80 \mu \mathrm{M}$ de L-ARG, o b) con $200 \mu \mathrm{M}$ de L-NAME. Se confeccionó una segunda curva dosisrespuesta a FE, en presencia de L-ARG o L-NAME. Al final del experimento, cada anillo fue pesado después de secarlo con papel filtro. La concentración de los fármacos se expresó como concentración final en el baño.

Análisis de los datos. Los datos fueron expresados como promedio \pm error estándar (ES). Los cambios de tensión por $\mathrm{KCl}$ se expresaron como $\mathrm{mg}$ de tensión desarrollada por cada anillo y los por FE, como porcentaje de la máxima tensión inducida por $\mathrm{KCl}$. El análisis estadístico de las curvas dosisrespuesta a FE antes y después de L-ARG o L-NAME fue hecho por el test de $t$ de Student para muestras pareadas y las diferencias entre los tres grupos de vasos fueron analizadas por ANOVA seguido del test de Student-Neuman-Keuls. La significancia estadística se aceptó al nivel de 5\% ( $p<0,05)$.

\section{ResUlTADOS}

Características de los pacientes. El perfil clínico de los pacientes incluidos comprende $38 \%$ con historia de infarto agudo del miocardio; 35\% con antecedentes de angina inestable y $55 \%$ de angina estable. Ningún paciente había sido sometido a cirugía coronaria previa y $17 \%$ tenía antecedentes de angioplastia.

Respuesta contráctil a $\mathrm{KCl}$. La respuesta contráctil a $80 \mathrm{mM}$ de $\mathrm{KCl}$-expresada en mg de tensiónpara cada tipo de vaso fue de $370 \pm 70$ para AMI, $1.400 \pm 150$ para VS y $2.350 \pm 410$ para AR $(p<0,05$ en todas las comparaciones).

Efecto de L-ARG. La exposición a $80 \mu \mathrm{M}$ de L-ARG durante 60 min no produjo cambio en la tensión basal de los segmentos arteriales o venosos.

Las curvas dosis-respuesta basales a FE generaron las siguientes tensiones máximas expresadas como porcentaje de la contracción inducida por $\mathrm{KCl}$ : AMI $91 \pm 15,2 \%$, VS $97,5 \pm 7,0 \%$ y AR que alcanzó un máximo significativamente menor de $65,5 \pm 9,0 \%(\mathrm{p}<0,05)$.

La incubación de los segmentos de AMI, AR y VS con L-ARG, determinó un desplazamiento de la curva dosis-respuesta a FE hacia la derecha, como puede observarse en la Figura 1, que se acompañó de una significativa disminución de la tensión máxima desarrollada con los siguientes valores $31,3 \pm 5,7 \%$ para IMA, $33 \pm 4,3 \%$ para RA y $54,2 \pm 6,3 \%$ para VS.

Efecto de L-NAME. La tensión basal de los anillos arteriales y venosos no se modificó con la exposición a $200 \mu \mathrm{M}$ de L-NAME, durante $60 \mathrm{~min}$.

La Figura 2 muestra que los valores de las curvas dosis-respuesta basales a $\mathrm{FE}$, expresadas como porcentaje de la contracción inducida por $\mathrm{KCl}$, alcanzan un máximo de 95,7士10,3\% para AMI y de $100 \pm 10 \%$ para VS. Sin embargo, para la AR se alcanzó un valor de $61 \pm 7,6 \%(p<0,05)$.

Las curvas dosis-respuesta obtenidas después de la incubación con $200 \mu \mathrm{M}$ de L-NAME presentaron un desplazamiento hacia la izquierda, acompañado de un significativo aumento de la tensión máxima cuyos valores relativos a $\mathrm{KCl}$ fueron de $122,5 \pm 11 \%$ para AMI, $128 \pm 12 \%$ para VS y $103 \pm 10,5 \%$ para AR.

\section{Discusión}

Los segmentos de vasos provenientes de pacientes portadores de cardiopatía coronaria, presentan respuesta contráctil a la administración de $\mathrm{KCl} 0$ 


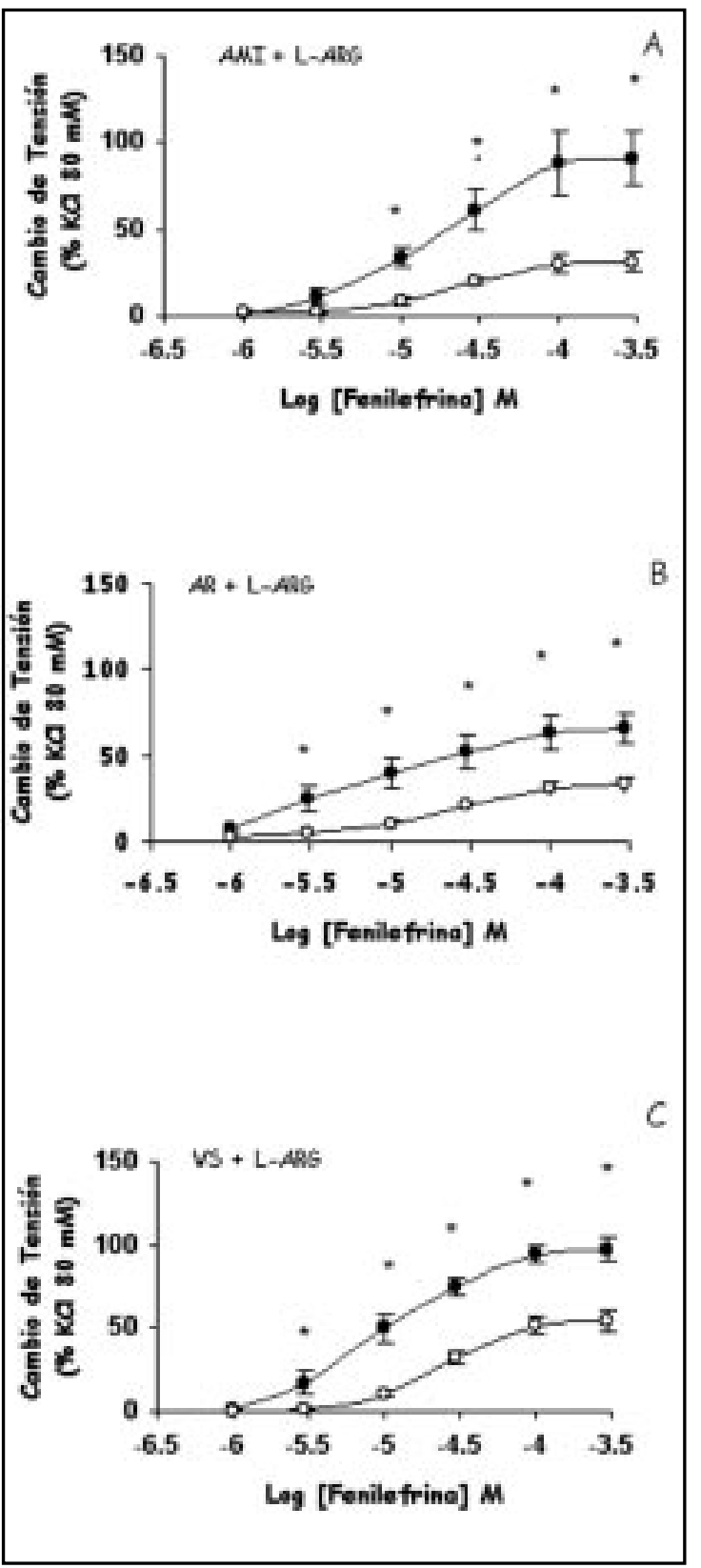

Figura 1. Efecto de L-arginina (L-ARG, $80 \mu \mathrm{M}$ ) en la tensión inducida por fenilefrina (FE) en anillos de arteria mamaria interna (AMI), panel A; en arteria radial (AR), panel B y en vena safena (VS), panel C. Los cambios se expresan en porcentaje de la respuesta máxima a $80 \mathrm{mM} \mathrm{KCl}$. Cada punto representa el promedio \pm ES de 6 a 7 experimentos. Curva dosis-respuesta antes ( $(\bullet)$ y después (O) de la incubación con L-ARG. ${ }^{*}=\mathrm{p}<0,05$.
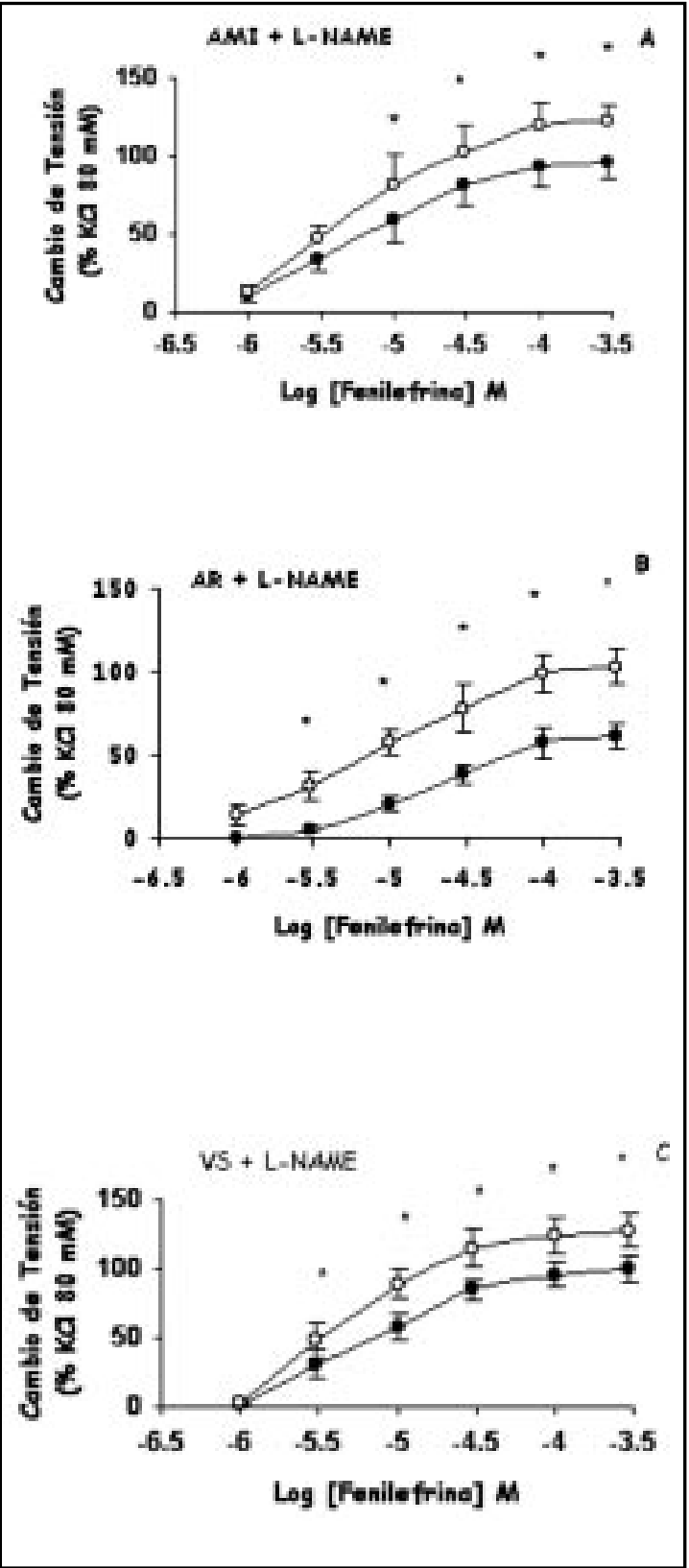

Figura 2. Efecto de $\mathrm{N}^{\mathrm{G}}$-nitro-L-arginina metil éster (LNAME, $200 \mu \mathrm{M}$ ) en la tensión inducida por fenilefrina (FE) en anillos de arteria mamaria interna (AMI), panel A; en arteria radial (AR), panel B y en vena safena (VS), panel C. Los cambios se expresan en porcentaje de la respuesta máxima a $80 \mathrm{mM} \mathrm{KCl}$. Cada punto representa el promedio \pm ES de 6 a 8 experimentos. Curva dosis-respuesta antes $(\bullet)$ y después $(0)$ de la incubación con L-NAME. $*=p<0,05$. 
FE, sin que se observe cambios en la tensión basal con la administración de L-NAME y L- ARG. Estudios previos muestran que la adición de LARG produce vasodilatación ${ }^{13}$ y la de L-NAME produce actividad contráctil 14,17,18, en los mismos tipos de vasos. Estas discordancias podrían deberse a las diferencias entre los protocolos utilizados, composición de la solución fisiológica salina, dosis de L-ARG o L-NAME, presencia de indometacina para inhibir síntesis de prostanoides 0 precontracción de los vasos en $20 \%$ del máximo efecto de $\mathrm{KCl}$.

Sin embargo, en concordancia con estudios previos $^{18}$, el pretratamiento con L-NAME, induce un mayor grado de contracción a FE en la AR ( $\Delta$ $42 \%)$ que en la VS $(\Delta 28 \%)$ y la AMI $(\Delta 26,8 \%)$. La incubación con L-ARG produce un menor grado de contracción a FE en AMI $(\Delta 60 \%)$, en VS $(\Delta$ $43 \%)$ y en $\operatorname{AR}(\Delta 33 \%)^{13}$.

El análisis de la contracción máxima basal desarrollada por la administración de FE, tanto en los conductos preincubados con L-NAME como con L-ARG, sugieren que en la AR existe una mayor capacidad basal de producción de NO, coincidente con otros estudios que le asignan a esta arteria una relativa mayor actividad de NOS, tanto en condiciones basales como por activación colinérgica comparada con los otros vasos ${ }^{17}$.

Además, el presente estudio está de acuerdo con los resultados obtenidos en el trabajo de Guzik y cols ${ }^{18}$, quienes usando los mismos segmentos de vasos, compararon la biodisponibilidad de NO, a través de la relajación inducida por acetilcolina y por la respuesta contráctil adicional a L-NAME en vasos precontraídos con FE. Esta última metodología es considerada un bioensayo de la biodisponibilidad de NO usando un método mecánico de tensión isométrica, más que un ensayo de agonismo farmacológico ${ }^{19}$.

Por otra parte, la contracción dosis-dependiente inducida por FE es potenciada por el pretratamiento con L-NAME, lo que es explicado por el bloqueo de la síntesis de NO. Si bien los resultados obtenidos en el presente trabajo no indican de manera directa que la producción de NO es mayor en la AR que en la AMI o VS, no se puede descartar una diferencia en la sensibilidad al NO. La menor contracción en presencia de L-ARG es explicada por el rol de precursor de la síntesis de NO propia de este aminoácido ${ }^{15}$.

Las arterias radial y mamaria interna poseen diferentes características anatómicas y fisiológicas $^{7}$. La AR tiene una gruesa capa muscular de aproximadamente $500 \mu \mathrm{m}$, en comparación a los cerca de $300 \mu \mathrm{m}$ que posee la $\mathrm{AMI}^{20}$. Estas diferencias podrían explicar la diferente respuesta contráctil a FE debido a los distintos niveles en la regulación de NOS, fenómeno que ya ha sido descrito para las diferencias encontradas en la producción basal de prostaciclinas, en estas arterias $^{6}$. De este modo se explicaría la variación en la actividad contráctil miogénica inducida por $\mathrm{KCl}$ en los diferentes vasos.

La integridad del endotelio y una adecuada producción de NO son elementos claves en la regulación vascular ${ }^{21}$. La reducción en la biodisponibilidad de NO está involucrada en el inicio, progresión y complicaciones de la ateroesclemsis 4 . Puesto que los vasos usados en este estudio provienen de pacientes con enfermedad coronaria demostrada, es razonable suponer que ellos podrían tener diferentes grados de disfunción endotelial de base, aunque en el presente trabajo no se midió directamente la relajación inducida por acetilcolina.

La disfunción endotelial progresa con la edad. El envejecimiento puede estar asociado con una menor disponibilidad de NO, lo cual puede conducir a una reducción marcada de la capacidad vasodilatadora (disfunción endotelial), asociada a la disminución de la producción de otros agentes vasodilatadores 0 al aumento de factores vasoconstrictores, tales como los relacionados con los receptores $\alpha$-adrenérgicos ${ }^{22}$.

En conclusión, el pretratamiento in vitro con L-ARG o con L-NAME, de anillos de AR, AMI o VS provenientes de cirugía de revascularización coronaria, mostraron diferencias en la tensión máxima inducida por FE en cada tipo de vaso, lo que podría estar relacionado con diferentes grados de producción endotelial de NO o diferencias en su sensibilidad.

Agradecimientos

Los autores agradecen a J. López, A. Correa, R. Zepeda y E. Prado por su eficiente asistencia técnica. 


\section{REFERENCIAS}

1. Dzimiri N, Chester AH, Aumen SP, Durán C, Yacoub $\mathrm{MH}$. Vascular reactivity of arterial comnary artery bypass grafts - Implications for their performance. Clin Cardiol 1996; 19: 165-71.

2. Miшs N. Arterial grafts for coronary artery bypass. Adv Card Surg 1997; 9: 195-216.

3. Davignon J, Ganz P. Role of endothelial dysfunction in atherosclerosis. Circulation 2004; 109: (Suppl. III), III-27 - III-32.

4. Luscher TF, Yang Z, Tschudi M, Von Segeser L, Stulz P, Boulanger $C$ et aL. Interaction between endothelin-1 and endothelium-derived relaxing factor in human arteries and veins. Cardiovasc Res 1990; 66: 1088-94.

5. Chester AH, Marchbank AJ, Borland JaA, Yacoub $\mathrm{MH}$, TAGGART DP. Comparison of the morphologic and vascular reactivity of the proximal and distal radial artery. Ann Thorac Surg 1988; 65: 1972-7.

6. Chardigny CI, Van Der Perre K, Simonet S, Descombes JJ, Fabiani JN, VerbeuRen TT. Platelets and prostacyclin in arterial bypasses: implications for coronary artery surgery. Ann Thorac Surg 2000; 69: 513-19.

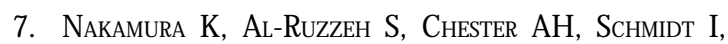
Barbir M, Yacoub MH, AmRanI M. Effects of cerivastatin on vascular function of human radial and left internal thoracic arteries. Ann Thorac Surg 2002; 73: 1860-5.

8. LANDMESSER U, HorNig B, DreXIER H. Endothelial function. A critical determinant in atherosclerosis. Circulation 2004; 109: [Suppl. II], II-27 - II-33.

9. Alderton WK, Cooper CE, Knowles RG. Nitric oxide synthases: structure, functions and inhibition. Biochem J 2001; 357: 593-615.

10. Palmer RJ, Rees DD, Ashton DS, Moncada S. Larginine is the physiological precursor for the formation of nitric oxide in endothelium-dependent relaxation. Biochem Biophys Res Commun 1988; 153: 1251-6.

11. LibBy P, RidKer PM, Maseri A. Inflammation and atherosclerosis. Circulation 2002; 105: 1135-43.
12. LauFs U. Beyond lipid-lowering effects of statins on endothelial nitric oxide. Eur J Clin Pharmacol 2003; 58: 719-31.

13. Valance P, Colier J, Moncada S. Nitric oxide synthesized from L-arginine mediates endothelium-dependent dilatation in human veins in vivo. Cardiovasc Res 1989; 23: 1053-7.

14. Rees DD, Palmer RMJ, Schulz R, Hodson HF, MONCADA S. Characterization of 3 inhibitors of endothelial nitric oxide synthase in vivo and in vitro. Br J Pharmacol 1990; 101: 746-52.

15. Böger RH, BOde-Böger SM. The clinical pharmacology of L-arginine. Annu Rev Pharmacol Toxicol 2001; 41: 79-99.

16. Pinardi G, Brieva C, López J, Correa A, Miranda HF. Alpha-1 adrenoceptor subtypes in the mesenteric vasculature and thoracic aorta of the rat. Pharmacol Rev Commun 1997; 9: 205-13.

17. Shapira OM, Xu A, Aldea GS, Vita JA, Shemin RJ, KEANEY JR JF. Enhanced nitric oxide-mediated vascular relaxation in radial artery compared with internal mammary artery or saphenous vein. Circulation 1999; 100: (Suppl. II), II-322 - II-327.

18. Guzik TJ, West NEJ, Pilai R, Taggart DP, Channon KM. Nitric oxide modulates superoxide release and peroxynitrite formation in human blood vessels. Hypertension 2002; 39: 1088-94.

19. Fleming I, Bauersachs J, Schafer A, Scholz D, AlderSHVILE J, BusSe R. Isometric contraction induces the $\mathrm{Ca}^{2+}$-independent activation of the endothelial nitric oxide synthase. Proc Natl Acad Sci USA 1999; 96: 1123-8.

20. Van Son JAM, Smedts F, Vincent JG, Van Lier HJJ, KUBAT K. Comparative anatomical studies of various arterial conduits for myocardial revascularization. J Thorac Cardiovasc Surg 1990; 99: 703-7.

21. Cable DG, Caccitolo JA, Pfeifer EA, Daly RC, Dearani JA, MuLan CJ et aL. Endothelial regulation of vascular contraction in radial and internal mammary arteries. Ann Thorac Surg 1999; 67: 1083-90.

22. Toro L, Marjic J, Nishimaru K, Tanaka Y, Song M, SteFAn E. Aging, ion channel expression and vascular function. Vasc Pharmacol 2002; 38: 73-80. 